\title{
The Phantom Divide in String Gas Cosmology
}

\author{
Brett McInnes \\ National University of Singapore \\ email: matmcinn@nus.edu.sg
}

\begin{abstract}
One of the main virtues of string gas cosmology is that it resolves cosmological singularities. Since the Universe can be approximated by a locally asymptotically de Sitter spacetime by the end of the inflationary era, a singularity theorem implies that these cosmologies effectively violate the Null Energy Condition [not just the Strong Energy Condition]. We stress that this is an extremely robust result, which does not depend on assuming that the spatial sections remain precisely flat in the early Universe. This means, however, that it must be possible for string cosmologies to cross the recently much-discussed phantom divide [from $\mathrm{w}<-1$ to $\mathrm{w}>-1$, where $\mathrm{w}$ is the equation-ofstate parameter]. This naturally raises the question as to whether the phantom divide can be crossed again, to account for recent observations suggesting that $\mathrm{w}<-1$ at the present time. We argue that non-perturbative string effects rule out this possibility, even if the NEC violation in question is only "effective".
\end{abstract}




\section{String Gases, Topology, and the Null Energy Condition}

The string gas cosmology of Brandenberger and Vafa [1] 2] 3as many attractions, but, among them, two stand out in particular: it is claimed that this approach can explain the existence of three large dimensions, and that it can abolish cosmological singularities ${ }^{1}$. In this work we shall investigate the precise meaning of this second statement, and explore the consequences.

In more detail, string gas cosmology is an attempt to use unconventional spacetime geometry - the spatial sections have the topology of a three-torus, in the simplest models - to obtain cosmological models in which the large dimensions are distinguished from the small by means of the concrete physical mechanism of string annihilation. Such cosmologies are generically non-singular, because there are momentum modes with energies quantised in multiples of $1 / R$, where $R$ is the radius of a given dimension in string units. In some sense which remains to be made precise, any attempt to probe length scales below the self-dual radius will turn out to be a probe of larger, T-dual scales. This is very desirable, but one would like to understand the consequences; it is reasonable to suppose that there are costs involved.

Because string gas cosmologies are fully non-singular, one must of course expect that some condition of the classical cosmic singularity theorem [11], page 266] is violated. Since all of the other conditions are certainly satisfied [genericity and, notably, the presence of compact edgeless achronal sets], the only possibility is that the Strong Energy Condition [SEC] is violated. This must be connected with an early period of inflation, which can in fact be achieved in string gas cosmology [12] 13]. As is well known, however [14, inflationary violations of the SEC in themselves by no means guarantee the avoidance of singularities; thus we need to investigate further.

A clue as to how to proceed is provided by the fact that, by the end of the inflationary era, the local geometry of the Universe closely resembles that of de Sitter spacetime; that is, from the point of view of still earlier eras, spacetime is asymptotically locally de Sitter towards the future. Combining this observation with the requirement that singularities should not appear, we find that string gas cosmologies effectively violate the Null Energy Condition [NEC], not just the SEC. This follows from the singularity theorem due to Andersson and Galloway [15]. The most remarkable feature of the theorem is that it allows us to reach this conclusion without making any assumptions about the detailed geometry of the early Universe. In fact, the crucial assumption is topological: it is that the spatial sections should have a topology of precisely the kind assumed in string gas cosmology. Thus, the theorem is ideally suited to this kind of cosmology; and, since the crucial condition is topological, the conclusion that the NEC has to be effectively violated is very robust - it does not depend on an assumed FRW structure or on the details of the Einstein equation.

In short, in string gas cosmology, a period of inflation has to be preceded by a period of [effective] NEC violation.

Before we proceed, let us be clear about the meaning of "effective" NEC violation. The Andersson-Galloway theorem actually implies a purely geometric conclusion, the

\footnotetext{
${ }^{1}$ See [4] [5] 6] 7] 8] for a sample of recent relevant work, and 9] [10] for clear summaries, many further references, and reports on recent advances on various problems connected with the theory.
} 
violation of the Null Ricci Condition [see below]. If the Einstein field equations hold exactly, then a violation of the Null Ricci Condition immediately implies a violation of the NEC, which is of course a condition on the stress-energy-momentum tensor. In more complex gravitational theories, the Einstein equations may not hold exactly; but one can still write the equations in the standard form, with an extra contribution to the "right hand side". In such cases a violation of the Null Ricci Condition can appear to cause a violation of the NEC, simply because [for example] the standard Friedmann equations are assumed in reconstructing the pressure of the dark energy from the spacetime geometry. In other words, the geometry of the spacetime will be such as would result from a violation of the NEC, even though no true matter field actually violates that condition. This is what we mean by "effective" NEC violation; it corresponds to a violation of the Null Ricci Condition.

All violations of the NEC, true or effective, are harder to account for theoretically than violations of the SEC [16]. The study of NEC-violating cosmologies was initiated in 17]; they are often singular [see [18] 19] for more recent references discussing the singular case], but the fact that they can be non-singular and asymptotically locally de Sitter was pointed out in [20], and this observation has been developed in various ways in [21] 22] 23] 24] 25] 26]. "Phantom cosmologies" involving true violations of the NEC encounter well-known objections [27, but these are avoided by more sophisticated models in which the violation is merely effective ${ }^{2}$ : for example, in brane-world theories [29] 30], or through quantum effects [31] 32, or by considering string-motivated Gauss-Bonnet corrections 33 to the Einstein-Hilbert Lagrangian. String theory apparently permits true NEC violation in the very early Universe; specifically, there are in fact explicit NECviolating cosmologies which have been constructed within string theory: Kachru and McAllister 34 have given such an example in the context of a warped deformed conifold in a Calabi-Yau compactification of II B string theory ${ }^{3}$. However, whether string theory allows NEC violations, even of the "effective" type, when the full non-perturbative theory is taken into account, remains an open question.

Returning to the case of string gas cosmology: the era of NEC violation we have been discussing must be very brief - it must come to an end and be replaced by an inflationary era [12] 13. Hence we should look for [true or effective] NEC violating effects which are evanescent. Notice too that if the inflationary era is governed by a dynamical inflaton as opposed to a pure cosmological constant] then it follows that it must be possible for these cosmologies to make the transition from $\mathrm{w}<-1$ to $\mathrm{w}>-1$, where $\mathrm{w}$ is the equationof-state parameter. [This question was first discussed in the context of the "phantom inflation" model [36] 37].] The ability of a cosmological model to "cross the phantom divide" in this way can be a very subtle matter, as has been emphasised recently in the astrophysics literature [38 39] 40] 41] 42] 43] 44]. [In fact, these works are concerned with a transition in the opposite direction, but the same issues arise here.] Thus string gas cosmology must belong to this special class of models. This is a highly non-trivial conclusion.

Next, in view of the fact that the Universe is again violating the SEC at the present

\footnotetext{
${ }^{2}$ See [28] for a general perspective on effective violations of the NEC.

${ }^{3}$ This is an effective scalar-tensor theory. The very subtle ways in which the NEC may or may not be violated in such theories is thoroughly explored in [35].
} 
time [45, it is natural to ask whether the same is true of the NEC: can the NEC, like the SEC, be noticeably violated over a lengthy period, including the present? Granted that string gas cosmologies are capable of crossing the "phantom divide" in one direction, it seems natural to suppose that they can cross in the other, from $\mathrm{w} \geq-1$ to $\mathrm{w}<$ -1. In fact, as $\mathrm{Hu}$ has recently stressed [41, such a crossing must occur if, as has been proposed [38, the observational data suggest that $\mathrm{w}$ is evolving rapidly. The question is far from academic, since a recent period of NEC violation is entirely compatible with the observational data [46]. Furthermore, some analyses of the data [47] suggest that they directly favour a recent crossing of the phantom divide. [However, other analyses, for example [48 [49] 50], suggest that the data are still compatible with a simple cosmological constant; the question remains open.]

Since we are now considering NEC violation over vast periods, it is clear that this second period of NEC violation must be of the "effective" kind, as for example arises very naturally in brane-world models [29] [30] $]^{4}$. We nevertheless wish to argue that nonperturbative string physics does not allow even effective violations of the NEC for an extended period, at least not in a way that could account for claimed observations of $\mathrm{w}$ $<-1$ at the present time. The relevant mechanism is the "Schwinger-like" production of brane-antibrane pairs, in the manner first discussed in 55] 53] [54] [55], and recently reconsidered in detail by Maldacena and Maoz [56] and others [57, 58, 59, 60] 61] 62]. The key property of this process that we need here is that it is determined entirely by the spacetime geometry: it does not "care" whether the NEC violation is true or effective. We show that if the NEC is violated in asymptotically locally de Sitter spacetimes - even if only effectively - then when a brane-antibrane pair is created, it is usually possible to move one member of the pair into a region where the action becomes negative. This is a signal of instability. The only way to avoid this instability is to choose the parameters so that the NEC-violating geometry approaches local de Sitter geometry extremely rapidly with the expansion of the Universe. This is precisely what we need in the earliest stages, to allow the transition to the inflationary era; but such a rapid approach to a de Sitterlike state would render this matter undetectable at the present time. In short, string cosmology predicts that the dark energy satisfies $w \geq-1$ at the present time, and that there has been no crossing of the phantom divide since the very earliest era.

We have based this discussion on string gas cosmology, but it is relevant to any asymptotically locally de Sitter cosmology with toral spatial sections; see for example 63 64 65]. For discussions of compact spatial sections in quantum cosmology, see [66 67 68 69], and see [70] for a discussion of the compatibility of compact spatial sections with inflation. Note that, in all these applications, including string gas cosmology, the spatial sections can be finite quotients of tori; in fact, some of these quotients are probably preferred to tori, since the internal space in the phenomenologically most interesting cases [71] [72] is a toral orbifold rather than a torus. See [73] for the interesting details of these quotients and [74] 62 for examples in which these details are physically important. For the sake of simplicity, however, we shall refer to all of these spaces as "tori", though "compact flat three-manifolds" would be more precise.

Before we proceed, we should clarify that neither this work nor any of those cited depends on the question [75] [76] as to whether a toral spatial structure is directly observable

\footnotetext{
${ }^{4}$ True NEC violation is also, however, possible on braneworlds: see [51 for a discussion.
} 
in, for example, the cosmic microwave background. A priori, one does not expect this to be possible, since the size of a flat torus is of course unrelated to its curvature and can be freely prescribed independently of all other parameters. An observation of toral structure would therefore constitute yet another "cosmic coincidence". In any case, our discussion has no bearing on this question.

Finally, our viewpoint on the whole question of NEC violation will be string-theoretic throughout. For other perspectives, see [77].

\section{The Global Formulation of String Gas Spacetimes}

In this section, we give a more precise discussion of the assertion that string gas cosmologies are non-singular. This will also permit us to state the Andersson-Galloway theorem.

A four-dimensional spacetime $\mathrm{M}_{4}$ with Lorentzian metric $g_{\mathrm{M}}$ is said [15] to have a regular future [past] spacelike conformal completion if $\mathrm{M}_{4}$ can be regarded as the interior of a spacetime-with-boundary $\mathrm{X}_{4}$, with a [non-degenerate] metric $g_{\mathrm{X}}$ such that the boundary is spacelike and lies to the future [past] of all points in $\mathrm{M}_{4}$, while $g_{\mathrm{X}}$ is conformal to $g_{M}$, that is, $g_{\mathrm{X}}=\Omega^{2} g_{\mathrm{M}}$, where $\Omega=0$ along the boundary but $\mathrm{d} \Omega \neq 0$ there. [There is a straightforward modification which allows us to define what it means for a spacetime to have a regular future and past spacelike conformal completion.] This definition captures

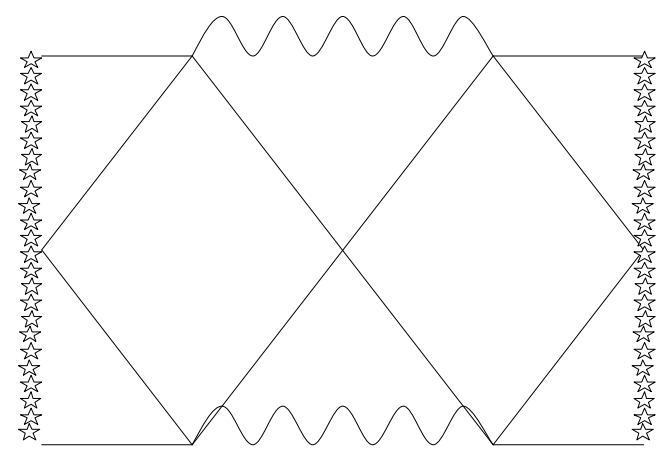

Figure 1: Schwarzschild-de Sitter According to Schwarzschild and de Sitter

one's intuitive understanding of an "asymptotically locally de Sitter" spacetime [towards the future, the past, or both]. For it is possible to show that the curvature of such a spacetime will increasingly resemble that of de Sitter spacetime along a timelike geodesic 
that escapes to future or past infinity. However, this is a strictly local statement; it is very important for us to understand how different such a spacetime can be from ordinary de Sitter spacetime.

An example will make the answer clear. Consider the Penrose diagram given in Figure 1. This spacetime is constructed as follows: first, recall that conventional de Sitter spacetime has the topology $\mathbb{R} \times \mathrm{S}^{3}$. We can however perform an identification of antipodal points on the three-sphere, to obtain a version with spatial sections isometric to the real projective space $\mathbb{R P ^ { 3 }}$. [This is in fact the way that de Sitter spacetime was interpreted by Schwarzschild and de Sitter — see [78] for a discussion of this, and [79] for further work in this direction.]

Now introduce an electrically neutral black hole into this version of de Sitter spacetime. The result is as shown in the diagram: the past and future singularities are shown as usual, together with the expected tunnel between two copies of what would otherwise have been the $\mathbb{R} \mathrm{P}^{3}$ version of de Sitter spacetime. The stars represent copies of the two-dimensional projective space $\mathbb{R \mathrm { P } ^ { 2 }}$; all other points represent $\mathrm{S}^{2}$.

This spacetime has a regular future and past spacelike conformal completion and so is asymptotically locally de Sitter by our definition. That is, any observer who does not fall into the black hole will eventually find that the geometry in his neighbourhood is indistinguishable from that of de Sitter spacetime. The global structure, however, could hardly be more different from that of de Sitter spacetime. Notice in particular that the conformal boundary in this case is certainly not $\mathrm{S}^{3}$ - indeed, it is not even compact, being four copies of an $\mathbb{R} \mathrm{P}^{3}$ from which a point has been deleted. [This non-compactness is closely related to the fact that the spacetime is singular, since this spacetime is globally hyperbolic - see [15].] In fact, the definition of "asymptotically locally de Sitter" allows for indefinite acceleration in a wide variety of ways. The definition does however exclude spacetimes like the Nariai spacetime [see for example [80] which fail to induce a nondegenerate conformal structure at infinity. This is justifiable on the grounds that such spacetimes are non-generic - two directions in Nariai spacetime do not expand at all, and hence they are "squeezed out" at infinity [78. The definition also excludes spacetimes like the ones considered recently by Freivogel and Susskind [81, where future infinity is not spacelike. These spacetimes accelerate but not indefinitely.

In short, the global structure of an asymptotically locally de Sitter spacetime can differ radically from that of de Sitter spacetime. We intend to make use of this freedom to investigate the global structure of string gas cosmologies.

A spacetime with a regular future [past] spacelike conformal completion is said to be future [past] asymptotically simple if every future [past] inextendible null geodesic has an endpoint on future [past] conformal infinity. This formalizes the idea of a non-singular asymptotically locally de Sitter spacetime - no photon is captured by a singularity in such a spacetime. The spacetime pictured in Figure 1 is neither future nor past asymptotically simple.

With these technicalities in hand, let us consider the global structure of a simple string gas cosmology.

In string gas cosmologies, it does not make sense for the cosmic scale factor to be smaller than a certain value: T-duality implies that attempts to probe below this value will reveal physics at the dual scale. In cosmology, this can be interpreted to mean that 
attempts to probe before a certain time will only produce data corresponding to a later time. Thus, spacetime should simply not exist for values of the scale factor corresponding to distances below the self-dual radius. We can model this as follows.

Let us begin with the more familiar case of de Sitter spacetime itself. This is not a string gas cosmology but its simplicity will clarify the point we wish to make. The metric, in terms of conformal time, together with the usual angular coordinates on the three-sphere of radius $\mathrm{L}$, is given by

$$
g\left(\mathrm{dS}_{4}\right)=\frac{\mathrm{L}^{2}}{\sin ^{2}(\eta)}\left[\mathrm{d} \eta^{2}-\mathrm{d} \chi^{2}-\sin ^{2}(\chi)\left\{\mathrm{d} \theta^{2}+\sin ^{2}(\theta) \mathrm{d} \phi^{2}\right\}\right] .
$$

Here $\eta$ is defined in the open interval $(0, \pi)$. Notice that, in this signature, this is a metric of constant negative curvature ${ }^{5}$; the cosmological constant takes the negative value $-3 / \mathrm{L}^{2}$.

Now perform an identification according to the isometry

$$
\Theta: \eta \rightarrow \pi-\eta
$$

The result is an orbifold spacetime in which the universe begins at the non-singular spacelike surface at $\eta=\pi / 2$, which is the distinguished surface corresponding to the smallest possible size permitted in this geometry. The universe can never be smaller than this, and there are no earlier events. This suggests a simple way of explicitly implementing the non-singularity of string gas cosmologies: the Universe should be "created from nothing" at this surface.

Notice that the initial surface is not embedded arbitrarily in the spacetime. The lower half of the Penrose diagram of ordinary de Sitter spacetime is an exact copy of the upper half, so that the surface $\eta=\pi / 2$ presents the same appearance from either side. The extrinsic curvature of the surface $\eta=\pi / 2$ [in the original spacetime, before the quotient is taken] is therefore zero, since otherwise it would provide a way of distinguishing one side from the other. The Penrose diagram is as shown in Figure 2 [in the case where the

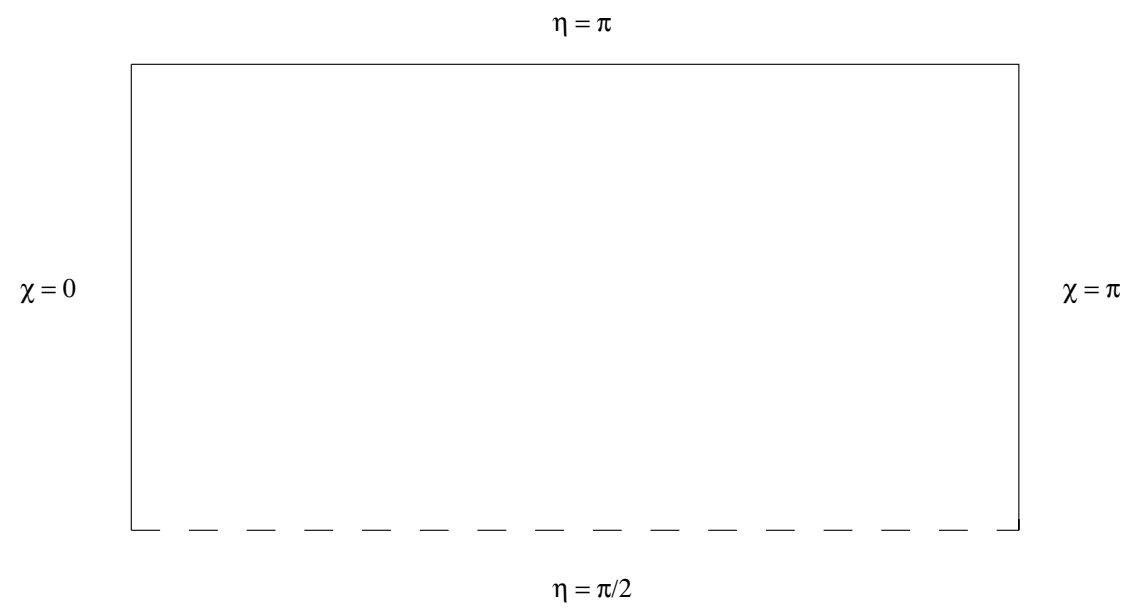

Figure 2: Penrose diagram of the de Sitter temporal orbifold.

\footnotetext{
${ }^{5}$ We are accustomed to thinking of de Sitter spacetime as having positive curvature, but this is merely a question of convention, depending on the choice of $(-+++)$ signature rather than $(+---)$.
} 
spatial topology is that of $\mathrm{S}^{3}$ rather than $\mathbb{R} \mathrm{P}^{3}$ - in the latter case it would be square]. The initial surface is at $\eta=\pi / 2$; it is located at a finite proper time prior to any other event in the spacetime - it is not a part of conformal infinity ${ }^{6}$. The latter is at $\eta=\pi$, and is of course spacelike; the spacetime has a regular future spacelike conformal completion. Notice that only one quarter of the events in this spacetime are ever visible to the observer at $\chi=0$, so this spacetime is indeed physically distinct from de Sitter spacetime. Notice too that conformal infinity is connected here, so the difficulties arising in connection with the disconnected conformal boundary of de Sitter spacetime are eliminated. [See [82] for a discussion of these issues.]

We use this example as the model for the formulation of the global structure of a true string gas cosmology. Assuming eternal acceleration, the spacetime should have a regular future spacelike conformal completion; to the past [at a finite proper time] there should be a boundary, so that the spacetime itself is a manifold-with-boundary having a connected boundary, and its conformal completion is a compact manifold-with-boundary having two boundary components. One should not think of either boundary as a physical object: the past boundary just represents the state of the Universe at the self-dual radius.

In the string gas case, there will be one crucial difference from the de Sitter case: the spatial sections will not be spherical, but flat. For simplicity we can take them to be modelled on a cubic torus. Such spacetimes are locally but not globally isotropic, so strictly speaking they cannot be represented on a Penrose diagram; however, we can inscribe any given cube in a sphere, and use these spheres to represent the corresponding cube. With this understanding, we can draw the Penrose diagram. Since the extent of conformal time is finite for an asymptotically locally de Sitter spacetime, the diagram is rectangular in shape. [If one embeds it in the Penrose diagram for Minkowski space, which can be done since all of the spacetimes here are locally conformally flat, the "rectangle" will bend down slightly at the top and bend inward on the right hand side, but of course these details do not affect anything we shall say.] The precise aspect ratio of the diagram, however, is not fixed classically. In the case of de Sitter spacetime, the shape is fixed by the fact that the size of the minimal three-sphere is determined by the value of the cosmological constant. Here, because the size of a torus is not related to its curvature, there is no such relation.

What does fix the aspect ratio? This can only be a matter of speculation at the moment, but some hints may be gleaned from the recent work of Bousso [83] on the difficulties which arise when one attempts to identify asymptotic observables in cosmology. As is well known, the existence of cosmological horizons in the asymptotically locally de Sitter case means that a certain proportion of the events in spacetime are forever invisible to an inertial observer, and this argues against the existence of asymptotic observables such as an S-matrix - though Bousso argues that other observables may be definable in certain cases. In the de Sitter case, shown in Figure 2, the proportion of invisible events is $3 / 4$. Notice however that the proportion would be reduced to $1 / 2$ if we had adopted the $\mathbb{R} \mathrm{P}^{3}$ interpretation of de Sitter spacetime, since the diagram is square in that case. In the string gas case, we can do even better, in fact arbitrarily better: by assuming that the rectangle is "tall and thin" [20] 84, we can reduce the fraction of permanently invisible events as much as we wish, since the top right-hand corner of the diagram will

\footnotetext{
${ }^{6}$ This is indicated by the dashed line in the diagram.
} 
have an arbitrarily small relative area in this case. Again, Bousso points out that even in a decelerating FRW model with non-compact flat spatial sections, there is at all times an infinitely large spatial region outside an observer's "causal diamond", so that the observer is permanently ignorant of an infinite amount of information stored there. This problem is solved very simply in the string gas case if the Penrose diagram is tall and thin: at all times the fraction of a spatial section contained in a causal diamond is non-zero, and, if the observer waits for a sufficiently long [but always finite] time, he will see an entire spatial section. He can then predict the fate of all objects in the Universe, including those ultimately destined to disappear beyond the horizon. In short, Bousso's arguments are in fact strong a priori arguments against infinite spatial sections, and in favour of the toral spatial structure assumed in string gas cosmology. They also suggest strongly that the Penrose diagram should be tall and thin.

An interesting possible shape for the Penrose diagram is given in Figure 3. The upper dot represents the present time, under the generally [but not universally [76] accepted assumption that the compactness of the spatial sections is not currently observable [85]; the horizontal line below it represents decoupling. The region below this horizontal line represents the combined eras of NEC violation, inflation, radiation dominance, and so on. Note that, in the case illustrated, an observer in the early Universe [represented by the lower dot] could detect the spatial compactness, in the sense that the same object is "visible" in two opposite directions. This feat will eventually be possible again, if we wait long enough.

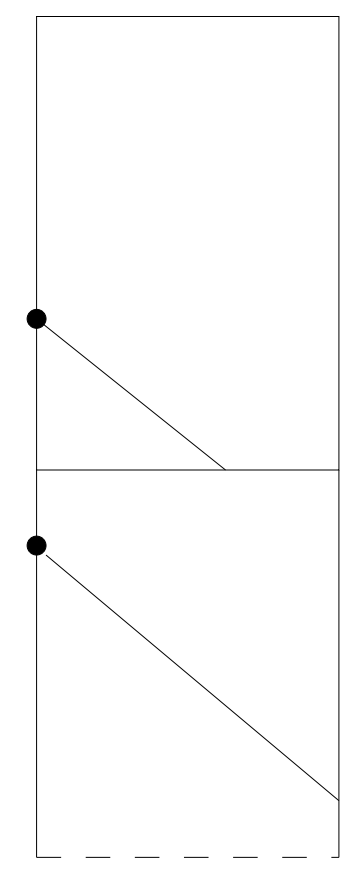

Figure 3: Penrose diagram of a string gas cosmology

We have stressed that the past boundary should not be regarded as a physical object: nothing "special" should happen there. By this we mean that T-duality should not obstruct attempts to probe distances below the self-dual radius, but rather should reveal them to be probes of larger distance scales - this should be the manner in which string gas cosmology smoothly resolves cosmological singularities. We can represent this in a 
formal way as follows: the embedding of the initial surface should be such that it is possible to glue an exact but "upside-down" copy of the Penrose diagram underneath the initial spacelike surface, in such a way that the transition is smooth.

The resulting Penrose diagram can be obtained simply by taking one like Figure 3 and "doubling" it by flipping it around the dashed line. It will formally resemble that of a "bounce" cosmology, in the sense that there are components of conformal infinity both at the bottom and at the top. However, the cosmology in fact does not bounce: time increases away from the dashed line in both directions ${ }^{7}$.

We regard this construction as a mere formal implementation of the idea that T-duality smoothly resolves cosmological singularities. The lower half of the "doubled" spacetime is really identical to the upper half; we have simply "unwrapped" an orbifold. However, there is an interesting analogy between this formal procedure and the global spacetime structure proposed by Carroll and Chen, in [86], as part of their theory of the arrow of time. There, too, one has a distinguished surface such that time increases away from it in both directions; in that case, however, it is not essential that the lower region be identical to the upper. It is nevertheless possible that true or effective NEC violations are generic also in the Carroll-Chen spacetime ${ }^{8}$.

Although the resemblance of this model to bouncing cosmologies is merely formal [so that, for example, we do not need to be concerned about the propagation of perturbations through the bounce], it nevertheless raises the same question that arises in connection with bounces: if the spatial sections are flat, the result could be an effective violation of all of the classical energy conditions. However, as this argument is based on assuming the precise form of the FRW metric [in particular, the exact flatness of the spatial sections at all times] and on the exact validity of the standard Friedmann equation, it is far from clear that it can be regarded as conclusive. We shall now show that the same conclusion can, however, be reached without making these assumptions.

First we remind the reader of the statement of the Null Energy Condition: this is simply the statement that for every null tangent vector $\mathrm{k}^{\mu}$ at every point of spacetime, the [total] stress-energy-momentum tensor $\mathrm{T}_{\mu \nu}$ should satisfy

$$
\mathrm{T}_{\mu \nu} \mathrm{k}^{\mu} \mathrm{k}^{\nu} \geq 0
$$

In the cosmological context, this just means that any observer of a cosmological spacetime with total energy density $\rho$ and pressure $\mathrm{p}$ should find that

$$
\rho+\mathrm{p} \geq 0
$$

is satisfied everywhere.

This is a very mild energy condition: it is implied by both the Weak and the Strong Energy Conditions, and it is satisfied both by de Sitter and by anti-de Sitter spacetimes. There is evidence [16] that it is essential for the functioning of the Covariant Entropy Bound [87], and severe instability problems can result if it is violated for ordinary matter fields. However, recall from the Introduction the distinction between "true" NEC violation

\footnotetext{
${ }^{7}$ This formal resemblance to a bounce cosmology is what allows us to escape the conclusions of [14, where contraction, even of this formal kind, is disallowed by assumption.

${ }^{8}$ The Carroll-Chen spacetime does not however have a regular future spacelike conformal completion, since the structure never completely settles down to a de Sitter-like state.
} 
[of the kind discussed in [27] ] and "effective" NEC violation, which arises from including all corrections to the Einstein field equations in the stress-energy-momentum tensor. In this case, we will find that the Universe evolves in a way which suggests that the NEC has been violated, even if this is not actually the case. To see how to detect this situation, we introduce the Null Ricci Condition [NRC], which requires that the Ricci tensor should satisfy

$$
\mathrm{R}_{\mu \nu} \mathrm{k}^{\mu} \mathrm{k}^{\nu} \geq 0 \text {. }
$$

for every null vector $\mathrm{k}^{\mu}$. Clearly the $\mathrm{NRC}$ is exactly equivalent to the NEC if the Einstein equations hold exactly. In other cases, however, a violation of the NRC means that the spacetime geometry is of the kind that would cause us to deduce NEC violation if we used the Einstein equations to try to compute the matter content from the geometry which is precisely what is done, for example, when observers of supernovae use the data to deduce apparent values of the dark energy equation-of-state parameter below -1 . That is, NRC violation is the formal interpretation of "effective" NEC violation.

We can now state the Andersson-Galloway singularity theorem [15]:

THEOREM [Andersson-Galloway]: Let $M_{4}$ be a globally hyperbolic spacetime with a regular future and past spacelike conformal completion. If the Null Ricci Condition is satisfied, and if the fundamental group of the Cauchy surfaces is infinite, then $M_{4}$ can be neither future nor past asymptotically simple.

In physical language: if a [past and future] asymptotically locally de Sitter spacetime is globally hyperbolic, has Cauchy surfaces with an infinite fundamental group, and satisfies the NRC [that is, it effectively satisfies the NEC], then it must be singular to the past and the future. In the spacetime pictured in Figure 1, the manifold is Einstein and so the NRC is satisfied. The spatial sections have the topology of the connected sum $\mathbb{R P}^{3}$ $\# \mathbb{R} \mathrm{P}^{3}$, which has an infinite fundamental group [it has the structure of a non-trivial $\mathrm{U}(1)$ bundle over $\left.\mathbb{R P}^{2}\right]$; thus the spacetime is forced to be singular by the topology of its spatial sections. Conversely, if we know that a spacetime with spatial sections of this kind is completely non-singular, then we can say that the topology of the spatial sections forces the spacetime to violate the $N R C$, that is, it enforces an effective violation of the NEC. Let us now see in detail how the Andersson-Galloway theorem applies to string gas cosmology.

Take a spacetime of the kind pictured in Figure 3, and "double" it, in the way we have described. This "doubled" spacetime will have a regular future and past spacelike conformal completion if the original spacetime had a regular future spacelike conformal completion. Similarly, the doubled space will be future and past asymptotically simple if the original spacetime was free of singularities. If the original spacetime is globally hyperbolic, so will be the doubled one. Similarly, a string gas cosmology has Cauchy surfaces which are either tori or finite quotients of tori, and so the same is true of the doubled spacetime. All such spaces have infinite fundamental groups. Combining all of these observations and applying the Andersson-Galloway theorem, we see that, as claimed, the NEC must effectively fail in the doubled spacetime, and so it must fail in the original spacetime.

It is noteworthy that the only part of this discussion which does not apply to de Sitter spacetime itself is the condition on the fundamental group of the spatial sections 
[which is finite for de Sitter spacetime and its relatives obtained by spatial topological identifications [78]. Thus de Sitter spacetime, which satisfies the NEC at all times, escapes our conclusion only because it has the wrong topology to be a string gas cosmology. We see that the distinctive spatial topology of string gas cosmology is at the heart of NEC violation, just as it is at the heart of the main advantages of string gas cosmology.

As with the classical Hawking-Penrose singularity theorems, the remarkable feature of the Andersson-Galloway theorem is that it allows us to avoid any symmetry assumptions. It also does not depend on the details of the Einstein equation. It is easy to show, using the Friedmann equations, that FRW cosmologies with exactly flat spatial sections can only "bounce" if the NEC is [at least effectively] violated, just as it is easy to see that all exact FRW cosmologies satisfying the SEC have to be singular. The point of the singularity theorems in both cases is that we can arrive at the same conclusion no matter what happens to the geometry of the spatial sections when the scale factor is small, and without using the Friedmann equations. The relevant behaviour in each case therefore cannot be avoided.

We conclude, then, that the avoidance of singularities in string gas cosmologies entails certain costs: the NEC is [at least effectively] violated in the earliest stages.

Just as a normal SEC-violating inflationary phase must come to an end and be supplanted by radiation and matter-dominated phases, so also the NEC-violating phase here must come to an end, making the transition to the inflationary phase. [An analogous statement holds true in the "phantom inflation" model [36] 37.] That is, expressed in terms of the equation-of-state parameter $\mathrm{w}$, the $\mathrm{w}<-1$ phase must be succeeded by a $\mathrm{w}>-1$ era, corresponding to the inflaton. But this crossing of the "phantom divide" is highly non-trivial, since it can lead to various unphysical effects [40. Indeed, it seems that no model based on a single scalar field can perform this feat. However, simple models with two scalar fields [41] 42] are capable of crossing the divide ${ }^{9}$. As Hu [41] stresses, such models can be regarded as a way of taking into account any hidden degrees of freedom in the dark energy [see also [43]. Since string gas cosmologies must be able to cross the divide, we conclude that they must be of this general kind. It is therefore highly interesting that the recent proposal of Biswas et al 13] for obtaining inflation in string gas cosmology does in fact involve two scalar fields, which however are not of the same kind as those considered by $\mathrm{Hu}$. It would be of great interest to combine these sets of ideas, to account for the pre-inflationary NEC-violating phase and its transition, crossing the phantom divide to the inflationary era, in the context of string gas cosmology.

Given that string gas cosmology must be able to cross the phantom divide in one direction, it is easy to imagine that it may cross it in the other - that is, in relatively recent times, $\mathrm{w}$ may have crossed from $\mathrm{w} \geq-1$ to $\mathrm{w}<-1$. This is in fact the kind of crossing discussed specifically in [41] and [42. The question, motivated by persistent observational suggestions that $\mathrm{w}$ may indeed currently be less than -1 [and also by analyses showing that better fits to the data [4] are obtained by models which cross the phantom divide in recent times], is whether string gas cosmologies can sustain NEC violation not just briefly, in the extremely early Universe, but also over cosmologically significant periods. We shall now argue that the answer is "no": there can be no second

\footnotetext{
${ }^{9}$ We stress however that these works are concerned with crossing the divide in the opposite direction to the one we are concerned with here.
} 
crossing of the divide according to string theory.

\section{NEC-Violating Cosmologies Unstable to Brane Pair Creation}

In this section, we shall attempt to understand the constraints imposed on an NECviolating cosmology by embedding it in string theory; this is reasonable, since string gas cosmologies are intrinsically stringy. Of course, the whole problem of deriving de Sitter spacetime from string theory is a formidable one. We have nothing to contribute to this question here; we shall simply assume that it can be done. Now de Sitter spacetime itself is not, of course, a realistic cosmology. The task of understanding the cosmic acceleration in string theory will only be complete after we have studied the back-reaction of various kinds of matter, including specifically stringy matter, on the spacetime geometry. In particular, the whole structure will be internally consistent only if we can demonstrate that a de Sitter background is not destabilized by stringy objects such as branes. As we shall see, this is no trivial requirement.

When the AdS/CFT correspondence is generalized away from Anti-de Sitter spacetime itself, there is a danger from various kinds of instability, perturbative and non-perturbative 61]. The particular effect with which we are concerned here involves the production, by means of a "Schwinger-like" pair-creation process, of BPS brane-antibrane pairs. This intrinsically non-perturbative process has been studied in depth in [52 [53] 554 [55] [56] [57 [58 [59 60 61] 62, and is well-understood in many cases, such as Einstein bulks with positively or negatively curved conformal boundaries. In the latter case, it is known that the pair-creation of branes is an unstable process.

We shall consider $(\mathrm{D}-1)$-branes in a space which generalizes $A d \mathrm{~S}_{\mathrm{D}+1}$ in some way. For the analysis of such generalized geometries, see [88; note that, as in this reference and in all of those given above, all of our discussions of brane physics refer to the Euclidean domain, so "AdS" means hyperbolic space with a specific kind of foliation. The nucleation of braneantibrane pairs in the Euclidean version will of course translate back to the Lorentzian domain in various ways, depending on the way in which the analytic continuation is done.

Although this is not always emphasised, asymptotically hyperbolic spaces can in fact be continued back to asymptotically locally de Sitter spacetimes [in the signature $(+-$ $--)$, which is why we have used that signature]. For example, if the hyperbolic space $\mathrm{H}^{4}$ is globally foliated by copies of $\mathrm{H}^{3}$, so that its metric is

$$
g\left(\mathrm{H}^{4}\right)=\mathrm{dt}^{2}+\cosh ^{2}(\mathrm{t} / \mathrm{L})\left[\mathrm{dr}^{2}+\mathrm{L}^{2} \sinh ^{2}(\mathrm{r} / \mathrm{L})\left\{\mathrm{d} \theta^{2}+\sin ^{2}(\theta) \mathrm{d} \phi^{2}\right\}\right],
$$

[see for example [89] then the continuation $r \rightarrow \mathrm{iL} \chi$ yields

$$
g\left(\mathrm{dS}_{4}\right)=\mathrm{dt}^{2}-\cosh ^{2}(\mathrm{t} / \mathrm{L}) \mathrm{L}^{2}\left[\mathrm{~d} \chi^{2}+\sin ^{2}(\chi)\left\{\mathrm{d} \theta^{2}+\sin ^{2}(\theta) \mathrm{d} \phi^{2}\right\}\right]
$$

and this is precisely the global de Sitter metric [equation (1), in different coordinates]. It has the same curvature $\left[=-1 / \mathrm{L}^{2}\right]$ as hyperbolic space, and also exactly the same isometry group [the orthogonal group $\mathrm{O}(1,4)$ ]. It is reasonable to assume that an asymptotically locally de Sitter spacetime with a radically unstable Euclidean version must itself be unstable. We shall not need to discuss the precise form this instability might take ${ }^{10}$.

\footnotetext{
${ }^{10}$ The obvious guess is that it involves "S-branes" 90 91 .
} 
Returning to the Euclidean case, the least-understood situation involves bulk manifolds which are not Einstein, and conformal boundaries which are scalar flat; even in the case of an Einstein bulk, the leading term in the action expansion given in [53] vanishes, and the outcome is determined by very complex higher-order terms. [See 61] for a discussion of these terms in this case.] But this least-understood case is, of course, precisely the case of most interest in cosmology, since cosmological spacetimes are almost never Einstein, and since the flat spatial sections of a physical FRW cosmology induce a flat structure at infinity. Thus it is not clear whether the kind of stringy instability we have been discussing can be avoided in the cosmological context; this is a fundamental question for any string cosmology.

It will be shown elsewhere 92 that if a cosmological model is asymptotically locally de Sitter towards the future, has flat spatial sections, and contains matter satisfying the NEC, then this non-perturbative instability cannot arise ${ }^{11}$. The question now is whether this satisfactory result is valid also in the case of string gas cosmology.

The brane action is, for a BPS brane,

$$
\mathrm{S}=\mathrm{T}\left(\mathrm{A}-\frac{\mathrm{D}}{\mathrm{L}} \mathrm{V}\right)
$$

where the bulk is $(\mathrm{D}+1)$-dimensional, $\mathrm{T}$ is the tension, $\mathrm{A}$ is the area, $\mathrm{V}$ the volume enclosed, and $\mathrm{L}$ is a constant length related to the asymptotic cosmological constant $\Lambda_{\infty}$ by

$$
\Lambda_{\infty}=-\mathrm{D} / \mathrm{L}^{2} .
$$

One sees at once that there is a danger that the volume term might dominate in some region of the bulk, in which case the nucleation of a brane-antibrane pair [a non-perturbative effect, since a barrier has to be overcome] would be an unstable process, since one could always move one member of the pair to the region where $S$ is negative. [See [56] for explicit examples where this happens, not necessarily near infinity.]

The essential point here is that the question as to which term dominates is a purely geometric one. The process does not "care" how we obtain a specific geometry - whether, for example, a specific shape is the result of "true" or "effective" violations of some energy condition. Once the metric is fixed, the question of the positivity of the brane action is determined. Henceforth, therefore, everything we say will hold for either kind of NEC violation.

In the specific case of the string gas geometry, as pictured in Figure 3, it is clear that the area term will dominate near to the initial surface; this is true whether or not the $\mathrm{NEC}$ is violated in that region. Thus the function $\mathrm{S}$ will be positive initially. The question is whether it remains positive.

Unfortunately there is no general theory regarding this question in the case of interest to us. Even in the case where the bulk is Einstein, the question of non-perturbative stability when the conformal boundary is flat is a subtle one, depending on certain constants [the "regularized area and volume" [61]] which one does not know how to evaluate in general. We can however investigate concrete examples, provided that we know the metric exactly, and we shall do this below. We shall first show very simply that there

\footnotetext{
${ }^{11}$ These spacetimes are singular, by the Andersson-Galloway theorem.
} 
does exist a large family of NEC-violating cosmologies which are unstable against brane pair creation. The issue is thus a very real one.

We shall be interested in cosmological models with metrics of the general form

$$
g^{-}=+\mathrm{dt}^{2}-\mathrm{A}^{2} \mathrm{a}(\mathrm{t})^{2}\left[\mathrm{~d} \theta_{1}^{2}+\mathrm{d} \theta_{2}^{2}+\mathrm{d} \theta_{3}^{2}\right]
$$

where the minus sign reminds us that we are in Lorentzian signature, where a $(\mathrm{t})$ is the scale factor, and where the spatial coordinates are angular coordinates on a cubic torus with all circumferences equal to $2 \pi \mathrm{A}$ on the initial surface [so that $\mathrm{a}(\mathrm{t})=1$ there]. This metric will resemble the de Sitter metric [in the flat slicing] at large t provided that the function $\mathrm{a}(\mathrm{t})$ has the appropriate [exponential] form there. That is, at large $\mathrm{t}$ the spacetime will closely resemble a spacetime of constant negative curvature. Following our discussion of de Sitter spacetime above, this means that the appropriate Euclidean version of this metric is simply

$$
g^{+}=+\mathrm{dt}^{2}+\mathrm{A}^{2} \mathrm{a}(\mathrm{t})^{2}\left[\mathrm{~d} \theta_{1}^{2}+\mathrm{d} \theta_{2}^{2}+\mathrm{d} \theta_{3}^{2}\right],
$$

since this metric approaches a metric of constant negative curvature for large $t$, that is, it is an asymptotically hyperbolic space. If one likes to think in terms of analytic continuation, one can move between the two versions by means of $\mathrm{A} \rightarrow \mathrm{iA}$.

It will be useful to describe the matter content of our NEC-violating models in the following way. We shall certainly want the local spacetime geometry to become indistinguishable from that of de Sitter space after the passage of a certain amount of time. To keep this to the fore, we shall write the total density and pressure as sums of the form

$$
\begin{aligned}
& \rho=\frac{3}{8 \pi \mathrm{L}^{2}}+\rho_{\psi}, \\
& \mathrm{p}=\frac{-3}{8 \pi \mathrm{L}^{2}}+\mathrm{p}_{\psi} .
\end{aligned}
$$

Obviously, it is always formally possible to write the energy density and the pressure in this way; the reader is free to regard this procedure as a mere formal device, or he may prefer to think in terms of some kind of more-or-less exotic "matter field" [labelled $\psi$, with density $\rho_{\psi}$ and pressure $\mathrm{p}_{\psi}$ ] propagating on a de Sitter background with cosmological constant $-3 / \mathrm{L}^{2}$, energy density $3 / 8 \pi \mathrm{L}^{2}$, and pressure $-3 / 8 \pi \mathrm{L}^{2}$. We do not necessarily regard this field as "matter" in the ordinary sense - it may simply parametrize the effects of higherorder curvature terms, of quantum effects [31]32, of brane-world physics, or other such contributions arising from the embedding of the system in higher-dimensional physics 29] 30] 28] 33] 34].

We are interested in determining whether an NEC-violating cosmology can be stable against brane pair-production. For clarity let us assume that the NEC is always violated. This means that $\rho+\mathrm{p}$ [equivalently, $\rho_{\psi}+\mathrm{p}_{\psi}$ ] is negative. Since a suitable combination of components of Einstein's equation for a spatially flat FRW cosmology yields

$$
\dot{\mathrm{H}}(\mathrm{t})=-4 \pi\left(\rho_{\psi}+\mathrm{p}_{\psi}\right)
$$

where $\mathrm{H}$ is the Hubble parameter and the dot denotes a cosmic time derivative, we see that $\mathrm{H}$ will always increase here. 
Now take equation (8) and evaluate it in the case of the Euclidean metric (11), obtaining

$$
\mathrm{S}(\mathrm{t})=\mathrm{T}\left[8 \pi^{3} \mathrm{~A}^{3} \mathrm{a}(\mathrm{t})^{3}-\frac{24 \pi^{3} \mathrm{~A}^{3}}{\mathrm{~L}} \int_{0}^{\mathrm{t}} \mathrm{a}(\tau)^{3} \mathrm{~d} \tau\right] .
$$

This expression is hard to handle, but the derivative is more informative: one has

$$
\dot{\mathrm{S}}(\mathrm{t})=24 \pi^{3} \mathrm{~T} \mathrm{~A}^{3} \mathrm{a}(\mathrm{t})^{3}\left[\mathrm{H}-\frac{1}{\mathrm{~L}}\right] .
$$

In our case $\mathrm{H}(0)=0$, and its asymptotic value in an asymptotically de Sitter spacetime is $1 / \mathrm{L}$; on the other hand, we saw above that $\mathrm{H}$ always increases in a spacetime which violates the NEC. It follows that $\mathrm{H}$ is always smaller than $1 / \mathrm{L}$, and hence that $\mathrm{S}(\mathrm{t})$ is a function which steadily decreases from its initial positive value of $8 \pi^{3} \mathrm{~T} \mathrm{~A}^{3}$. It could still be asymptotic to a positive value, however.

The right side of equation (16) contains a product of $\mathrm{a}(\mathrm{t})^{3}$, which increases without limit in an asymptotically de Sitter spacetime, with $\mathrm{H}-1 / \mathrm{L}$, which tends to zero. Simple calculus now implies that

$$
\lim _{\mathrm{t} \rightarrow \infty} \dot{\mathrm{S}}(\mathrm{t})=-8 \pi^{3} \mathrm{TA}^{3} \lim _{\mathrm{t} \rightarrow \infty}\left[\mathrm{a}(\mathrm{t})^{3} \dot{\mathrm{H}} / \mathrm{H}\right]
$$

and now equation (14) gives

$$
\lim _{\mathrm{t} \rightarrow \infty} \dot{\mathrm{S}}(\mathrm{t})=32 \pi^{4} \mathrm{TA}^{3} \mathrm{~L} \lim _{\mathrm{t} \rightarrow \infty}\left[\mathrm{a}(\mathrm{t})^{3}(\rho+\mathrm{p})\right],
$$

which can also be usefully written as

$$
\lim _{\mathrm{t} \rightarrow \infty} \dot{\mathrm{S}}(\mathrm{t})=32 \pi^{4} \mathrm{TA}^{3} \mathrm{~L} \lim _{\mathrm{t} \rightarrow \infty}\left[\mathrm{a}(\mathrm{t})^{3}\left(\rho_{\psi}+\mathrm{p}_{\psi}\right)\right]
$$

Since $\rho+\mathrm{p}$ and $\rho_{\psi}+\mathrm{p}_{\psi}$ are negative, it is clear that if the limit on the right is a non-zero constant or diverges, then the derivative $\dot{S}(\mathrm{t})$ either approaches a constant negative value or diverges to negative infinity; in either case $\mathrm{S}(\mathrm{t})$ will not only be eventually negative but also unbounded below. We conclude that these cosmologies will definitely be unstable to brane pair production if $\rho_{\psi}+\mathrm{p}_{\psi}$ tends to zero more slowly than $a(t)^{-3}$.

We have derived this conclusion without making any assumptions other than that the NEC is violated [in either sense]. We shall now see that far stronger conclusions can be reached in the context of specific spacetime geometries.

\section{Brane Pair Production: Constant EOS Parameter}

In the previous section we saw that the NEC-violating matter has to dilute rapidly in order to avoid instability due to brane pair production. The condition we derived was, however, merely a necessary one for stability: it is not clear that it is sufficient. With the help of specific examples, we shall now argue that it is not sufficient. Ultimately the real question is whether it is at all possible to avoid this problem, and, if so, at what cost.

In order to proceed, we need to make some assumptions about $\rho_{\psi}$ and $\mathrm{p}_{\psi}$; these will be as general and as conservative as possible.

In early discussions of phantom cosmology, concern was often expressed that the phantom energy might propagate outside the light-cone. This is understandable: in phantom 
cosmologies one can always find an observer who observes a spacelike energy-momentum vector for the phantom matter. However, the situation is not as simple as it seems, because there is more than one way to build a spacelike energy-momentum vector.

We shall distinguish between two cases, which we call "semi-exotic" and "fully exotic". In the first case, the energy-momentum vector of the $\psi$ field alone is timelike but pastpointing. This kind of matter is "semi-exotic" in the sense that while a past-pointing energy-momentum vector is of course unorthodox, at least there can be no question of the energy of the $\psi$ matter propagating outside the light cone. [Of course we do not need to think of energy "propagating backwards in time" here, any more than one speaks of ordinary energy "propagating" forwards in time.] In terms of $\rho_{\psi}$ and $\mathrm{p}_{\psi}$, we are requiring that $\mathrm{p}_{\psi}$ should be related to $\rho_{\psi}$ [which is negative] by

$$
\mathrm{p}_{\psi}=\mathrm{w}_{\psi} \rho_{\psi}
$$

where $\mathrm{w}_{\psi}$, the equation-of-state parameter for $\psi$ alone, satisfies $-1<\mathrm{w}_{\psi}<1$ so that the energy-momentum vector for $\psi$ alone remains timelike. The point now is that the energy-momentum four-vector of the entire system will be spacelike if we insert a small amount of this "semi-exotic" field into de Sitter spacetime, simply because the energymomentum four-vector of the constant dark energy is null and future-pointing, and the sum of a "small" past-pointing timelike four-vector with a future-pointing null vector will be spacelike. This achieves a violation of the NEC [corresponding to a total energymomentum four-vector which is spacelike] without any propagation of energy outside the light-cone, since the kind of dark energy represented by a cosmological constant cannot "propagate" - its energy density is rigidly constant in space and time. By contrast, if the $\psi$ field itself [assumed not to exhibit this "rigidity"] already has a spacelike energymomentum vector, then we are in the "fully exotic" case, and causality may be a real issue.

We assume for simplicity that $\mathrm{w}_{\psi}$ does not vary with time. With this assumption one can solve the Einstein equation for a metric of the form (10), obtaining the exact solution [20]:

$$
g^{-}(\gamma, \mathrm{A}, \mathrm{L})=+\mathrm{dt}^{2}-\mathrm{A}^{2} \cosh ^{\left(\frac{4}{\gamma}\right)}\left(\frac{\gamma \mathrm{t}}{2 \mathrm{~L}}\right)\left[\mathrm{d} \theta_{1}^{2}+\mathrm{d} \theta_{2}^{2}+\mathrm{d} \theta_{3}^{2}\right]
$$

where $\gamma$ is a constant given by

$$
\gamma=3\left[1+\mathrm{w}_{\psi}\right]
$$

Clearly this geometry can be interpreted in the way we have suggested: that is, we can regard the $t<0$ part of the geometry as a mere copy of the $t>0$ part; the surface $t=0$ is the orbifold surface at which the Universe begins. The conformal boundary is connected, compact, spacelike, and flat, that is, the conformal structure can be represented by a flat metric.

The requirement that the energy-momentum vector of the $\psi$ field should be timelike is here expressed by

$$
0<\gamma<6
$$

so the value 6 marks the boundary between the semi-exotic and fully exotic cases. This metric is asymptotically locally de Sitter towards the future: the scalar curvature is given by

$$
\mathrm{R}\left(g^{-}(\gamma, \mathrm{A}, \mathrm{L})\right)=-\frac{12}{\mathrm{~L}^{2}}+\frac{3}{\mathrm{~L}^{2}}(4-\gamma) \operatorname{sech}^{2}\left(\frac{\gamma \mathrm{t}}{2 \mathrm{~L}}\right)
$$


with an asymptotic value of $-12 / \mathrm{L}^{2}$, in agreement with the de Sitter value from equation (11) [with signature as given]. The NEC is violated at all times in this spacetime: one has

$$
\rho_{\psi}+\mathrm{p}_{\psi}=\frac{-\gamma}{8 \pi \mathrm{L}^{2}} \operatorname{sech}^{2}\left(\frac{\gamma \mathrm{t}}{2 \mathrm{~L}}\right)
$$

Notice that the NEC-violating matter is diluted towards $t=\infty$, and is concentrated near $\mathrm{t}=0$. In fact we have

$$
\left|\rho_{\psi}+\mathrm{p}_{\psi}\right|_{\text {Max }}=\frac{\gamma}{8 \pi \mathrm{L}^{2}}
$$

so $\gamma$ measures the degree to which the NEC is violated; as expected, the violation is less severe in the preferred, "semi-exotic" case. On the other hand, the value of t when this quantity falls to half of its maximal value, $\mathrm{t}_{1 / 2}$, is given by

$$
\mathrm{t}_{1 / 2}=\frac{2 \mathrm{~L}}{\gamma} \cosh ^{-1}(\sqrt{2})
$$

so we see that the NEC-violating "matter" dies off away from $\mathrm{t}=0$ more slowly when $\gamma$ is small. Large values of $\gamma$ correspond to an NEC-violating phase which is intense but short-lived. For an application involving lengthy intervals of time, this suggests that small values of $\gamma$ are preferred.

The Penrose diagram of this spacetime is rectangular, with width determined by A and height determined by $\mathrm{L}$ and $\gamma$ : the height is in fact

$$
\int_{0}^{\infty} \frac{\mathrm{dt}}{\cosh ^{\left(\frac{2}{\gamma}\right)}\left(\frac{\gamma \mathrm{t}}{2 \mathrm{~L}}\right)},
$$

which is always larger than L, and which becomes arbitrarily large for sufficiently small $\gamma$. In view of our previous discussion of "tall and thin" Penrose diagrams, this is further evidence that it would be best to remain in the "semi-exotic" regime of small values for $\gamma$. Unfortunately we shall now see that this is not possible.

We can write

$$
\rho_{\psi}+\mathrm{p}_{\psi}=\frac{-\gamma}{8 \pi \mathrm{L}^{2}} \mathrm{a}(\mathrm{t})^{-\gamma}
$$

Comparing this with equation (19), we see that this model will be unstable against the production of brane pairs if $\gamma$ takes any value less than or equal to 3 . Thus for example equation (21) with $\gamma=2$,

$$
g^{-}(2, \mathrm{~A}, \mathrm{~L})=+\mathrm{dt}^{2}-\mathrm{A}^{2} \cosh ^{2}\left(\frac{\mathrm{t}}{\mathrm{L}}\right)\left[\mathrm{d} \theta_{1}^{2}+\mathrm{d} \theta_{2}^{2}+\mathrm{d} \theta_{3}^{2}\right]
$$

represents a spacetime which is strongly unstable against brane pair creation, the brane action being unbounded below. This is the first known explicit example of a spacetime which is unstable in the Seiberg-Witten [53] sense despite not having a negatively curved conformal boundary. Notice that it differs from de Sitter spacetime only in that the spatial sections are flat and compact instead of spherical. The introduction of NECviolating matter into de Sitter spacetime allows us to flatten its spatial sections, but at the cost of rendering it strongly unstable.

While the general argument of the preceding section does not apply to these spacetimes when $\gamma$ exceeds 3, it is intuitively clear that many of them will also have action functions 
$\mathrm{S}_{\gamma}(\mathrm{t})$ which become [and therefore remain] negative. In fact, in this case we can settle this by a direct calculation. Substituting a(t) from equation (21) into equation (15), we have

$$
\mathrm{S}_{\gamma}(\mathrm{t})=\mathrm{T}\left[8 \pi^{3} \mathrm{~A}^{3} \cosh ^{(6 / \gamma)}\left(\frac{\gamma \mathrm{t}}{2 \mathrm{~L}}\right)-\frac{24 \pi^{3} \mathrm{~A}^{3}}{\mathrm{~L}} \int_{0}^{\mathrm{t}} \cosh ^{(6 / \gamma)}\left(\frac{\gamma \tau}{2 \mathrm{~L}}\right) \mathrm{d} \tau\right]
$$

This can be evaluated explicitly in many cases of interest: for example, in the "borderline" case $\gamma=3$ [which we know to be unstable] we have

$$
\mathrm{S}_{3}(\mathrm{t})=4 \pi^{3} \mathrm{~A}^{3} \mathrm{~T}\left[1+\mathrm{e}^{-3 \mathrm{t} / \mathrm{L}}-\frac{3 \mathrm{t}}{2 \mathrm{~L}}\right]
$$

which is indeed unbounded below.

Since we understand the case $\gamma \leq 3$, let us assume that $\gamma>3 / 2$. Then for large $\mathrm{t}$ we can approximate $\cosh ^{(6 / \gamma)}\left(\frac{\gamma \mathrm{t}}{2 \mathrm{~L}}\right)$ by the first two terms in its Taylor expansion. Doing this, we find after extensive simplifications that, approximately,

$$
\mathrm{S}_{\gamma}(\mathrm{t}) \approx 2^{\left(3-\frac{6}{\gamma}\right)} \pi^{3} \mathrm{~A}^{3} \mathrm{~T}\left[\frac{6}{\gamma-3} \mathrm{e}^{(3-\gamma) \mathrm{t} / \mathrm{L}}-\frac{(6-\gamma)(3+\gamma)}{\gamma(\gamma-3)}\right]
$$

where we can exclude $\gamma=3$ since we have already treated that case exactly. In agreement with our general result, we find that if $\gamma$ is slightly less than 3 , then the exponential term dominates and is negative, so $\mathrm{S}_{\gamma}(\mathrm{t})$ is unbounded below. The new result is that, if $\gamma$ is larger than 3 but [in accordance with (23) above] less than 6 , then the constant term dominates and is negative. In the limit the approximation becomes exact, and we have, for any $\gamma>3$,

$$
\lim _{t \rightarrow \infty} S_{\gamma}(t)=-2^{\left(3-\frac{6}{\gamma}\right)} \pi^{3} A^{3} \mathrm{~T} \frac{(6-\gamma)(3+\gamma)}{\gamma(\gamma-3)} .
$$

Thus in this case $\mathrm{S}_{\gamma}(\mathrm{t})$ will decline to a negative value. At any point it can always be made smaller, by moving to larger values of $t$; nevertheless, in this case it is bounded below [by its limit]. We see however that the pair-production instability is present for all of our "semi-exotic" models.

It is of course true that the right side of (34) can be made arbitrarily close to zero in magnitude by taking $\gamma$ sufficiently close to 6 . Then $\mathrm{S}_{\gamma}(\mathrm{t})$ will still become negative, but only at a huge value of $t$. In this way, one can postpone the onset of instability until the remote future, at the cost of a severe fine-tuning.

The alternative is to ignore the inequalities (23) and allow the $\psi$ field to have a spacelike energy-momentum vector; in this "fully exotic" case, $\mathrm{S}_{\gamma}(\mathrm{t})$ certainly remains positive everywhere, but one has to be concerned about the possible propagation of energy outside the light cone. Nevertheless, such possibilities have been seriously considered 93, and we do not rule them out completely, at least not in the very early Universe. In short, it $i s$ possible to keep the brane action positive everywhere, but only by means of choosing rather extreme parameter values.

Interesting as all these possibilities may be, however, the key point is this: if $\gamma$ is substantially larger than 3, then our theory cannot account for any observations corresponding to a value of $w<-1$ at the present time. For if $\gamma$ is so large, we see from (29) that the NEC-violating field decays away extremely rapidly with the growth of the Universe, far more rapidly than either relativistic or non-relativistic matter, and of course 
far faster than cosmological constant-style dark energy, quintessence, or an inflaton. This is exactly what we want in the very early Universe, to ensure a crossing of the phantom divide so that inflation can proceed; but it is not what we want if we are trying to account for purported observations of phantom behaviour at the present time. In fact, to account for such observations without interfering [for example] with the standard nucleosynthesis computations, we would need $\gamma$ to be very small, far below 3 , and we know that this is out of the question.

To summarize, we have found that string theory only tolerates NEC violation if the deviation from a pure positive cosmological constant decays extremely rapidly: specifically, it must [according to equation (29)] decay according to at least the inverse sixth power of the scale factor. This is highly desirable if we are describing the NEC-violating phase which, according to string gas cosmology, must precede inflation - the NEC violation decays very rapidly and allows inflation to proceed in the way described in [12] or [13. However, it also means that string theory predicts that there can be no appreciable deviation of the dark energy equation-of-state parameter below -1 at the present time. This conclusion holds even if the NEC "violation" is of the "effective" kind.

\section{Specific Potentials}

Thus far, we have not tried to be more precise about the nature of the $\psi$ field; we saw that this is not really necessary in order to draw our conclusions. Now, however, that we know that NEC violation is relevant only in the extremely early Universe, for a very short time, we can allow ourselves to consider more specific models for the $\psi$ field. As is well known [17, the simplest possible parametrization of it is as a scalar field with a kinetic term of the "wrong" sign. It is also well known [27] that this particular kind of phantom model is subject to quantum instabilities, but perhaps this is of less concern for a model which does not attempt to violate the NEC over a cosmologically significant period. In any case, we simply note here that if we introduce an explicit $\psi$ field of this kind, then the precise spacetime [20] we discussed above [with metric $\left.g^{-}(\gamma, \mathrm{A}, \mathrm{L})\right]$ can be obtained from the surprisingly simple potential

$$
\mathrm{V}_{\gamma}(\psi)=\frac{\gamma-6}{16 \pi \mathrm{L}^{2}} \cos ^{2}(\sqrt{2 \pi \gamma} \psi)
$$

Bear in mind here that this potential is to be superimposed on a de Sitter background, so the claim is that $g^{-}(\gamma, \mathrm{A}, \mathrm{L})$ solves the Friedmann equation when the total density is the sum of $3 / 8 \pi \mathrm{L}^{2}$, the negative kinetic term for $\psi$, and $\mathrm{V}_{\gamma}(\psi)$. One can also verify using the field equation for $\psi$ that, with this potential, $\mathrm{w}_{\psi}$ is indeed constant, as we assumed. [Notice that $\mathrm{V}_{\gamma}(\psi)$ is positive when $\gamma$ exceeds 6 , and this is indeed precisely the condition

for the magnitude of the pressure, $\frac{1}{2} \dot{\psi}^{2}+\mathrm{V}_{\gamma}(\psi)$ for a scalar with a reversed kinetic term, to exceed the magnitude of the density, $\frac{1}{2} \dot{\psi}^{2}-\mathrm{V}_{\gamma}(\psi)$.] A two-scalar model, consisting of a $\psi$-field with a potential similar to (35), together with a more conventional inflaton field, could give a simple quantitative description of the crossing of the phantom divide in the early Universe. [See [94] for a recent discussion of related models in the context of bouncing cosmologies.]

The reader may object that our strongest conclusions depend on the assumption that $\mathrm{w}_{\psi}$ is independent of time - this corresponds to the specific potential (35) and the exact 
metric (21). This is of course true, though it is hard to believe that a relaxation of this assumption can materially change our conclusions. Nevertheless it is desirable to check our results with an exact phantom model having a non-constant $\mathrm{w}_{\psi}$. In fact, an exact phantom cosmological solution has recently become available [95] in which $\mathrm{w}_{\psi}$ is not constant, and it is very interesting to examine that solution from this point of view.

Aref'eva, Koshelev, and Vernov consider a potential of the form

$$
\mathrm{V}_{\mathrm{AKV}}(\psi)=\frac{1}{2 \mathrm{~L}^{2}}\left[1-\psi^{2}\right]^{2}+\frac{1}{12 \mathrm{M}_{\mathrm{p}}^{2} \mathrm{~L}^{2}} \psi^{2}\left[3-\psi^{2}\right]^{2}
$$

where $\mathrm{L}$ is a constant length which sets the scale of the potential at the initial time, and where $\mathrm{M}_{\mathrm{p}}$ is a gravitational coupling constant. [Note that small $\mathrm{M}_{\mathrm{p}}$ corresponds to strong coupling.] This theory is a sort of truncation of the one we have been considering above; it has several remarkable properties, including the existence of an exact solution for a metric of the form (10), with the scale function given by

$$
\mathrm{a}(\mathrm{t})_{\mathrm{AKV}}=\cosh ^{1 /\left(3 \mathrm{M}_{\mathrm{p}}^{2}\right)}(\mathrm{t} / \mathrm{L}) \exp \left[\frac{1}{12 \mathrm{M}_{\mathrm{p}}^{2}} \tanh ^{2}(\mathrm{t} / \mathrm{L})\right]
$$

This spacetime can be interpreted as a string gas cosmology, with the initial surface at $\mathrm{t}$ $=0$. It is asymptotically locally de Sitter with asymptotic cosmological constant

$$
\Lambda_{\mathrm{AKV} \infty}=-1 /\left(3 \mathrm{M}_{\mathrm{p}}^{4} \mathrm{~L}^{2}\right)
$$

The total energy density and pressure are given 95] by

$$
\begin{gathered}
\rho_{\mathrm{AKV}}=\frac{1}{12 \mathrm{M}_{\mathrm{p}}^{2} \mathrm{~L}^{2}} \tanh ^{2}(\mathrm{t} / \mathrm{L})\left[3-\tanh ^{2}(\mathrm{t} / \mathrm{L})\right]^{2} \\
\mathrm{p}_{\mathrm{AKV}}=-\frac{1}{\mathrm{~L}^{2}} \operatorname{sech}^{4}(\mathrm{t} / \mathrm{L})-\frac{1}{12 \mathrm{M}_{\mathrm{p}}^{2} \mathrm{~L}^{2}} \tanh ^{2}(\mathrm{t} / \mathrm{L})\left[3-\tanh ^{2}(\mathrm{t} / \mathrm{L})\right]^{2} .
\end{gathered}
$$

These expressions split very naturally according to the pattern of (12) and (13); we can regard the phantom matter here as a combination of a de Sitter cosmological constant with a negative-density field:

$$
\begin{gathered}
\rho_{\mathrm{AKV}}=\frac{1}{3 \mathrm{M}_{\mathrm{p}}^{2} \mathrm{~L}^{2}}-\frac{1}{12 \mathrm{M}_{\mathrm{p}}^{2} \mathrm{~L}^{2}} \operatorname{sech}^{4}(\mathrm{t} / \mathrm{L})\left[3+\operatorname{sech}^{2}(\mathrm{t} / \mathrm{L})\right] \\
\mathrm{p}_{\mathrm{AKV}}=-\frac{1}{3 \mathrm{M}_{\mathrm{p}}^{2} \mathrm{~L}^{2}}+\operatorname{sech}^{4}(\mathrm{t} / \mathrm{L})\left[-\frac{1}{\mathrm{~L}^{2}}+\frac{1}{12 \mathrm{M}_{\mathrm{p}}^{2} \mathrm{~L}^{2}}\left[3+\operatorname{sech}^{2}(\mathrm{t} / \mathrm{L})\right]\right] .
\end{gathered}
$$

Hence, in our notation, the equation-of-state parameter of the negative-density field is

$$
\mathrm{w}_{\psi \mathrm{AKV}}=-1+\frac{12 \mathrm{M}_{\mathrm{p}}^{2}}{3+\operatorname{sech}^{2}(\mathrm{t} / \mathrm{L})},
$$

and we see at once that $\mathrm{w}_{\psi}$ AKV does indeed vary with time, increasing steadily from its minimum value at $\mathrm{t}=0$ to its maximum at infinity,

$$
\mathrm{w}_{\psi \operatorname{AKV}}(\max )=-1+4 \mathrm{M}_{\mathrm{p}}^{2}
$$


We see from (43) that $\mathrm{w}_{\psi} \mathrm{AKV}$ never falls below -1 , approaching it only in the limit where $\mathrm{M}_{\mathrm{p}}$ tends to zero - note that this is the limit of strong coupling of the phantom to gravity, but it is also the limit of small asymptotic cosmological constant. If the theory is to remain "semi-exotic", that is, for the energy-momentum four-vector of the $\psi$ field is to remain timelike, then $\mathrm{w}_{\psi \mathrm{AKV}}(\max )$ must not exceed unity, and so we must have

$$
\mathrm{M}_{\mathrm{p}}^{2}<1 / 2
$$

that is, the coupling has to be at least this strong in the semi-exotic case.

Now let us investigate the stability of these cosmologies against brane pair creation. Substituting (37), (41) and (42) into (18) we find that the derivative of the brane action in this case satisfies

$$
\lim _{\mathrm{t} \rightarrow \infty} \dot{\mathrm{S}}_{\mathrm{AKV}}\left(\mathrm{M}_{\mathrm{p}}^{2} ; \mathrm{t}\right)=-32 \pi^{4} \mathrm{TA}^{3} \mathrm{~L}^{-1} \exp \left(\frac{1}{4 \mathrm{M}_{\mathrm{p}}^{2}}\right) \lim _{\mathrm{t} \rightarrow \infty} \cosh ^{\left[\mathrm{M}_{\mathrm{p}}^{-2}-4\right]}(\mathrm{t} / \mathrm{L}),
$$

so clearly we must have

$$
\mathrm{M}_{\mathrm{p}}^{2}>1 / 4
$$

if the brane action itself, $\mathrm{S}_{\mathrm{AKV}}\left(\mathrm{M}_{\mathrm{p}}^{2} ; \mathrm{t}\right)$, is to have any hope of remaining positive.

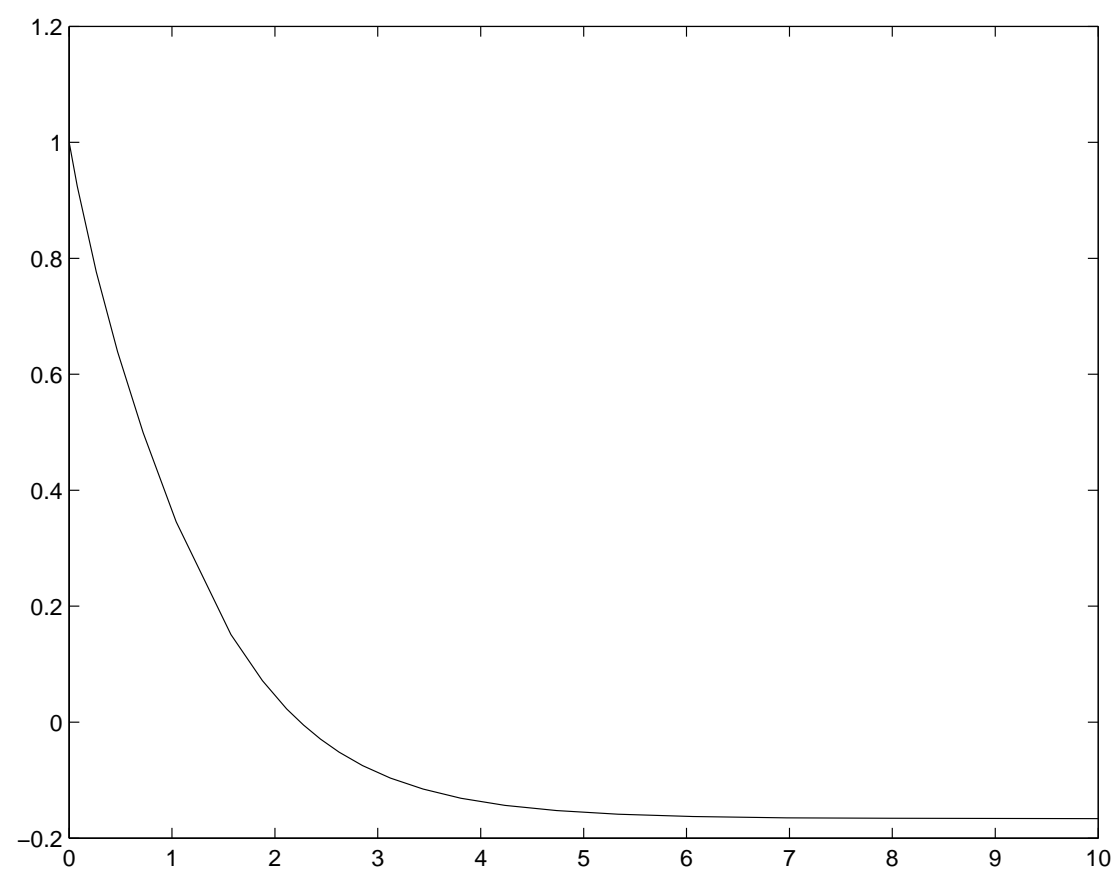

Figure 4: The action $\mathrm{S}_{\mathrm{AKV}}(1 / 3 ; \mathrm{t})$.

In short, $\mathrm{M}_{\mathrm{p}}^{2}$ must in any case be at least $1 / 4$; between $1 / 4$ and $1 / 2$ the non-de Sitter contribution to the energy-momentum vector will be timelike; beyond $1 / 2$, the contribution will be spacelike. While we would therefore prefer values corresponding to the "semi-exotic" regime, our experience in the preceding section leads us to expect that, while values between $1 / 4$ and $1 / 2$ will lead to an action which is bounded below, the action will nevertheless become negative in this case. 
The brane action in this case is

$$
\begin{aligned}
\mathrm{S}_{\mathrm{AKV}}\left(\mathrm{M}_{\mathrm{p}}^{2} ; \mathrm{t}\right)= & 8 \pi^{3} \mathrm{~A}^{3} \mathrm{~T}\left\{\cosh ^{1 /\left(\mathrm{M}_{\mathrm{p}}^{2}\right)}(\mathrm{t} / \mathrm{L}) \exp \left[\frac{1}{4 \mathrm{M}_{\mathrm{p}}^{2}} \tanh ^{2}(\mathrm{t} / \mathrm{L})\right]\right. \\
& \left.-\frac{3}{\mathrm{~L}} \int_{0}^{\mathrm{t}} \cosh ^{1 /\left(\mathrm{M}_{\mathrm{p}}^{2}\right)}(\tau / \mathrm{L}) \exp \left[\frac{1}{4 \mathrm{M}_{\mathrm{p}}^{2}} \tanh ^{2}(\tau / \mathrm{L})\right] \mathrm{d} \tau\right\},
\end{aligned}
$$

which of course has to be studied numerically. For simplicity we choose the parameters so that $\mathrm{L}=8 \pi^{3} \mathrm{~A}^{3} \mathrm{~T}=1$, so that $\mathrm{S}_{\mathrm{AKV}}\left(\mathrm{M}_{\mathrm{p}}^{2} ; 0\right)=1$ for all choices of $\mathrm{M}_{\mathrm{p}}^{2}$ - other choices will change the details of the graphs of $\mathrm{S}_{\mathrm{AKV}}\left(\mathrm{M}_{\mathrm{p}}^{2} ; \mathrm{t}\right)$ but not their basic shape.

We have made numerous trials with values of $\mathrm{M}_{\mathrm{p}}^{2}$ in various ranges, and the results confirm our conjecture: for example, Figure 4 shows the action function in a typical "semiexotic" case, $\mathrm{M}_{\mathrm{p}}^{2}=1 / 3$; we see that the action does indeed become negative. Thus, as in the case where the $\psi$ field has a constant equation-of-state parameter, we cannot take refuge in the semi-exotic regime: the only way to keep the brane action positive is to go to the "fully exotic" situation in which the non-de Sitter part of the energy-momentum vector is itself already spacelike. Again, numerical trials show that this does work: see for

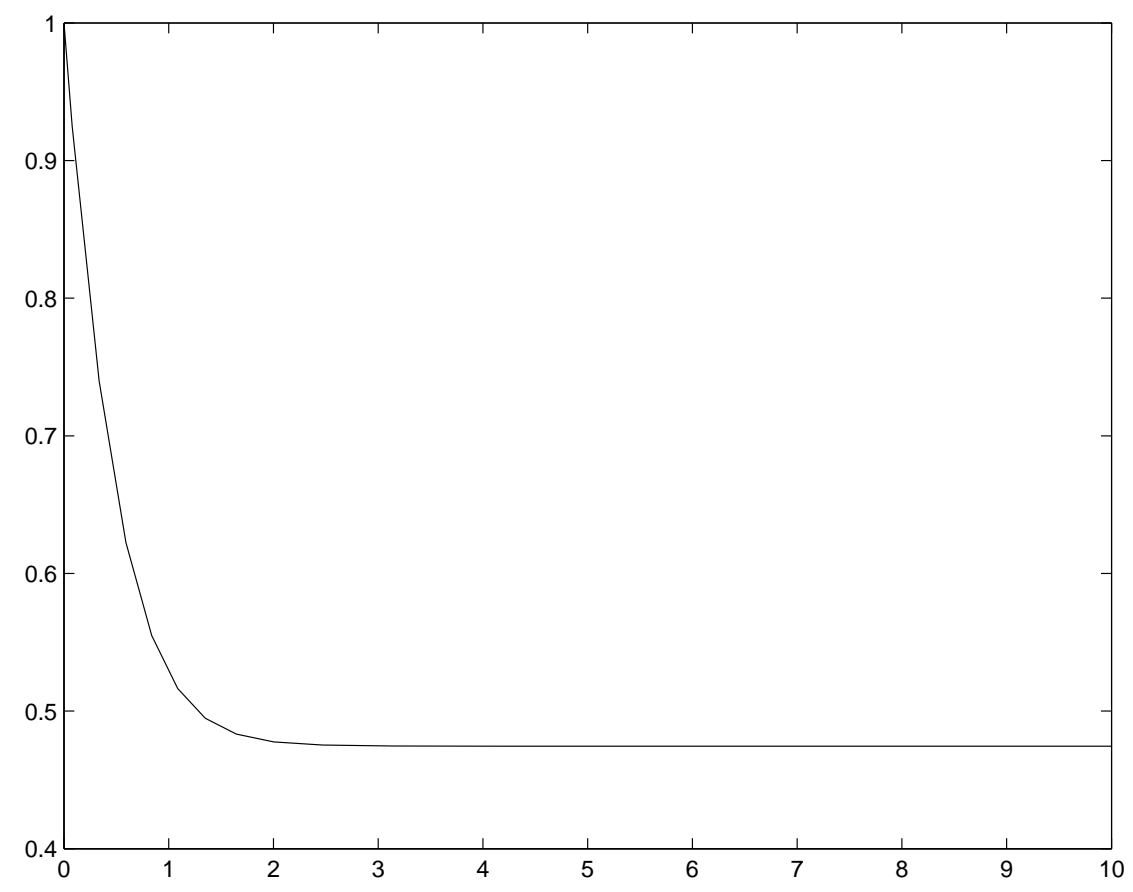

Figure 5: The action $\mathrm{S}_{\mathrm{AKV}}(1 ; \mathrm{t})$.

example Figure 5, which displays the action function when $\mathrm{M}_{\mathrm{p}}^{2}=1$. Thus the situation is the same whether or not we fix the equation-of-state parameter of the non-de Sitter contribution to be a constant. We believe that this conclusion holds for any non-singular phantom cosmological model: brane pair-production instability can only be avoided in the "fully exotic" case, corresponding to extremely fast decay of the non-de Sitter component as the Universe expands.

\section{Conclusion}

We have three major conclusions. 
First, string gas cosmologies must violate the NEC, at least effectively, in their early histories, and this conclusion is very robust: ultimately it is due to the topology of the spatial sections.

Second, this means that string gas cosmologies must be able to cross the phantom divide, so models of inflation in string theory must be compatible with such crossings; understanding this will no doubt require a careful analysis of the internal degrees of freedom in the dark energy generally. Of course, one has to ask whether, in any case, an NEC-violating phase provides suitable initial conditions for inflation; but this is not the place to discuss that intricate question.

Thirdly, we saw that if a certain non-perturbative string effect is taken into account, NEC violation in string gas cosmology can only occur over brief periods: the NECviolating "field" [which need not correspond to a true matter field] must dilute extremely rapidly with the expansion of the Universe. This is just what we want if we wish to account for $\mathrm{w}<-1$ in the extremely early Universe, but it is not compatible with any such observation in the relatively recent past. The phantom divide can be crossed only once: the theory predicts that more precise observations of [for example] supernovae will eventually indicate a dark energy equation-of-state parameter $\mathrm{w} \geq-1$. See [50] for the prospects for this; note meanwhile that since it can be argued that the current data [46] actually seem to favour $\mathrm{w}<-1$, this is not a trivial statement.

The most striking aspect of this part of our discussion was that it applies whether the NEC is violated in the true sense or only effectively. The particular kind of unstable production of brane pairs which is relevant here is sensitive only to the spacetime geometry - the way in which that geometry is shaped [whether by reversed-sign kinetic terms in a scalar field lagrangian, or by brane-world models in which all matter fields actually satisfy the NEC] is quite irrelevant. Hence our conclusion here - that, according to string gas cosmology, current indications of $\mathrm{w}<-1$ must be misleading — is robust.

The unusual features of string gas cosmology considered here stem from the fact that these spacetimes are not singular. One naturally wonders whether brane pair creation instability can be a problem even in more conventional, initially singular cosmologies. We shall investigate this question elsewhere.

We began this investigation with a discussion of the Andersson-Galloway theorem [15], which is clearly the basic result on the spacetime structure of string gas cosmologies. One of the basic assumptions of the theorem, however, is that the spacetime in question is asymptotically locally de Sitter. This is a reasonable assumption in the context of inflationary cosmology, but it is not obvious that it is relevant to the current phase of cosmic acceleration. It is entirely possible that at some point in the relatively near future, the current acceleration will cease, and be replaced by a contraction, leading to a Big Crunch, as discussed in [96] 56] 58 [59 60] 62]; see also [97] for the observational position. Note that the models considered in [58, 59] 62. have flat compact spatial sections, as in string gas cosmology. However, of course, a string gas cosmology cannot have a Crunch, any more than it can have a Bang. It would be interesting to understand the details of the way in which string gas cosmologies avert a Crunch. 


\section{Acknowledgements}

The author is extremely grateful to Wanmei for the diagrams and for unwavering support through difficult times. He would also like to acknowledge the kind hospitality of the High Energy, Cosmology, and Astroparticle Physics Section of the Abdus Salam International Centre for Theoretical Physics, where much of the work described here was done.

\section{References}

[1] R. H. Brandenberger and C.Vafa, Superstrings in the Early Universe, Nucl.Phys.B 316 (1989) 391

[2] S. Alexander, R. Brandenberger, D. Easson, Brane Gases in the Early Universe, Phys.Rev. D62 (2000) 103509, arXiv:hep-th/0005212

[3] Robert Brandenberger, Damien A. Easson, Dagny Kimberly, Loitering Phase in Brane Gas Cosmology, Nucl.Phys. B623 (2002) 421, arXiv hep-th/0109165

[4] Scott Watson, Robert Brandenberger, Linear Perturbations in Brane Gas Cosmology, JHEP 0403 (2004) 045, arXiv:hep-th/0312097

[5] Thorsten Battefeld, Scott Watson, Effective Field Theory Approach to String Gas Cosmology, JCAP 0406 (2004) 001, arXiv:hep-th/0403075

[6] Richard Easther, Brian R. Greene, Mark G. Jackson, Daniel Kabat, String windings in the early universe, arXiv hep-th/0409121

[7] Rebecca Danos, Andrew R. Frey, Anupam Mazumdar, Interaction Rates in String Gas Cosmology, Phys.Rev. D70 (2004) 106010, arXiv:hep-th/0409162

[8] Antonio Campos, Asymptotic cosmological solutions for string/brane gases with solitonic fluxes, arXiv:hep-th/0501092

[9] Robert Brandenberger, Yeuk-Kwan E. Cheung and Scott Watson, Moduli Stabilization with String Gases and Fluxes, arXiv:hep-th/0501032

[10] Subodh P. Patil, Robert H. Brandenberger, The Cosmology of Massless String Modes, arXiv:hep-th/0502069

[11] S. W. Hawking, G. F. R. Ellis, The Large Scale Structure of Space-Time, Cambridge University Press, 1973.

[12] Robert Brandenberger, Damien A. Easson, Anupam Mazumdar, Inflation and Brane Gases, Phys.Rev. D69 (2004) 083502, arXiv:hep-th/0307043

[13] Tirthabir Biswas, Robert Brandenberger, Damien A. Easson, Anupam Mazumdar, Coupled Inflation and Brane Gases, arXiv/hep-th/0501194

[14] Arvind Borde, Alan H. Guth, Alexander Vilenkin, Inflation is not past-eternal, Phys.Rev.Lett. 90 (2003) 151301, arXiv:gr-qc/0110012 
[15] L. Andersson, G.J. Galloway, dS/CFT and spacetime topology, Adv.Theor.Math.Phys. 6 (2003) 307, arXiv:hep-th/0202161

[16] Brett McInnes, The Covariant Entropy Bound, Brane Cosmology, and The Null Energy Condition, JHEP 0212(2002)053, arXiv:hep-th/0212014

[17] R.R. Caldwell, A Phantom Menace? Cosmological consequences of a dark energy component with super-negative equation of state, Phys.Lett. B545 (2002) 23, arXiv:astro-ph/9908168

[18] Shin'ichi Nojiri, Sergei D. Odintsov, Shinji Tsujikawa, Properties of singularities in (phantom) dark energy universe, arXiv:hep-th/0501025

[19] Yi-Huan Wei, Big Rip in SO(1,1) phantom universe, arXiv gr-qc/0502077

[20] Brett McInnes, The dS/CFT Correspondence and the Big Smash, JHEP 08 (2002) 029, arXiv:hep-th/0112066

[21] Shin'ichi Nojiri, Sergei D. Odintsov, Quantum escape of sudden future singularity, Phys.Lett. B595 (2004) 1, arXiv:hep-th/0405078

[22] Sushil Srivastava, Future Universe with w $<-1$ Without Big Smash, arXiv:astro-ph/0407048

[23] Puxun Wu, Hongwei Yu, Avoidance of Big Rip In Phantom Cosmology by Gravitational Back Reaction, arXiv:astro-ph/0407424

[24] Shin'ichi Nojiri, Sergei D.Odintsov, The final state and thermodynamics of dark energy universe, Phys.Rev. D70 (2004) 103522, arXiv:hep-th/0408170

[25] Yi-Huan Wei, Late-time phantom universe in $\mathbf{S O}(\mathbf{1}, \mathbf{1})$ dark energy model with exponential potential, arXiv gr-qc/0410050

[26] Ruben Curbelo, Tame Gonzalez, Israel Quiros, Interacting Phantom Energy and Avoidance of the Big Rip Singularity, arXiv:astro-ph/0502141

[27] Sean M. Carroll, Mark Hoffman, Mark Trodden, Can the dark energy equation-of-state parameter w be less than -1?, Phys.Rev. D68 (2003) 023509, arXiv:astro-ph/0301273

[28] Emilio Elizalde, Shin'ichi Nojiri, Sergei D. Odintsov, Peng Wang, Dark Energy: Vacuum Fluctuations, the Effective Phantom Phase, and Holography, arXiv:hep-th/0502082

[29] Varun Sahni, Yuri Shtanov, Braneworld models of dark energy, JCAP 0311 (2003) 014, arXiv:astro-ph/0202346

[30] Varun Sahni, Cosmological Surprises from Braneworld models of Dark Energy, arXiv astro-ph/0502032 
[31] V.K. Onemli, R.P. Woodard, Super-Acceleration from Massless, Minimally Coupled $\phi^{4}$, Class.Quant.Grav. 19 (2002) 4607, arXiv:gr-qc/0204065

[32] V. K. Onemli, R. P. Woodard, Quantum effects can render $\mathrm{w}<-1$ on cosmological scales, Phys.Rev. D70 (2004) 107301, arXiv gr-qc/0406098

[33] Shinji Tsujikawa, Robert Brandenberger, Fabio Finelli, On the Construction of Nonsingular Pre-Big-Bang and Ekpyrotic Cosmologies and the Resulting Density Perturbations, Phys.Rev. D66 (2002) 083513, arXiv:hep-th/0207228

[34] S. Kachru, L. McAllister, Bouncing Brane Cosmologies from Warped String Compactifications, JHEP 0303 (2003) 018, arXiv:hep-th/0205209

[35] Emilio Elizalde, Shin'ichi Nojiri, Sergei D. Odintsov, Late-time cosmology in (phantom) scalar-tensor theory: dark energy and the cosmic speed-up, Phys.Rev. D70 (2004) 043539, arXiv hep-th/0405034

[36] Yun-Song Piao, E Zhou, Nearly Scale-Invariant Spectrum of Adiabatic Fluctuations May be from a Very Slowly Expanding Phase of the Universe, Phys.Rev. D68 (2003) 083515, arXiv:hep-th/0308080

[37] Yun-Song Piao, Yuan-Zhong Zhang, Phantom Inflation and Primordial Perturbation Spectrum, Phys.Rev. D70 (2004) 063513, arXiv:astro-ph/0401231

[38] Ujjaini Alam, Varun Sahni, Tarun Deep Saini, A. A. Starobinsky, Is there Supernova Evidence for Dark Energy Metamorphosis?, Mon.Not.Roy.Astron.Soc. 354 (2004) 275, arXiv:astro-ph/0311364

[39] Bo Feng, Xiulian Wang, Xinmin Zhang, Dark Energy Constraints from the Cosmic Age and Supernova, Phys.Lett. B607 (2005) 35, arXiv astro-ph/0404224

[40] Alexander Vikman, Can dark energy evolve to the Phantom?, Phys.Rev. D71 (2005) 023515, arXiv:astro-ph/0407107

[41] Wayne Hu, Crossing the Phantom Divide: Dark Energy Internal Degrees of Freedom, Phys.Rev. D71 (2005) 047301, arXiv:astro-ph/0410680

[42] Robert R. Caldwell, Michael Doran, Dark-Energy Evolution Across the CosmologicalConstant Boundary, arXivastro-ph/0501104

[43] Hao Wei, Rong-Gen Cai, Hessence: A New View of Quintom Dark Energy, arXiv:hep-th/0501160

[44] Xiao-fei Zhang, Hong Li, Yun-Song Piao, Xinmin Zhang, Two-field Models of Dark Energy with Equation of State Across -1, arXiv:astro-ph/0501652

[45] Adam G. Riess et al, Type Ia Supernova Discoveries at z $>1$ From the Hubble Space Telescope: Evidence for Past Deceleration and Constraints on Dark Energy Evolution, Astrophys.J. 607 (2004) 665, arXiv:astro-ph/0402512 
[46] Amol Upadhye, Mustapha Ishak, Paul J. Steinhardt, Dynamical dark energy: Current constraints and forecasts, arXiv:astro-ph/0411803

[47] L. Perivolaropoulos, Constraints on linear-negative potentials in quintessence and phantom models from recent supernova data, arXiv astro-ph/0412308

[48] T. Roy Choudhury, T. Padmanabhan, Cosmological parameters from supernova observations: A critical comparison of three data sets, Astron.Astrophys. 429 (2005) 807, arXiv:astro-ph/0311622

[49] H.K.Jassal, J.S.Bagla, T.Padmanabhan, WMAP constraints on low redshift evolution of dark energy, Mon.Not.Roy.Astron.Soc. 356 (2005) L11, arXiv:astro-ph/0404378

[50] Yun Wang, Max Tegmark, Uncorrelated Measurements of the Cosmic Expansion History and Dark Energy from Supernovae, arXiv astro-ph/0501351

[51] Gianluca Calcagni, Patch dualities and remarks on nonstandard cosmologies, Phys.Rev. D71 (2005) 023511, arXiv:gr-qc/0410027

[52] J. Maldacena, J. Michelson, and A. Strominger, Anti-de Sitter Fragmentation, JHEP 9902 (1999) 011, arXiv:hep-th/9812073

[53] Nathan Seiberg, Edward Witten, The D1/D5 System And Singular CFT, JHEP 9904 (1999) 017, arXiv:hep-th/9903224

[54] Edward Witten, S.-T. Yau, Connectedness Of The Boundary In The AdS/CFT Correspondence, Adv.Theor.Math.Phys. 3 (1999) 1635, arXiv:hep-th/9910245

[55] Mingliang Cai, Gregory J. Galloway, Boundaries of Zero Scalar Curvature in the AdS/CFT Correspondence, Adv.Theor.Math.Phys. 3 (1999) 1769, arXiv:hep-th/0003046

[56] Juan Maldacena, Liat Maoz, Wormholes in AdS, JHEP 0402 (2004) 053, arXiv:hep-th/0401024

[57] Alex Buchel, Gauge theories on hyperbolic spaces and dual wormhole instabilities, Phys.Rev. D70 (2004) 066004, arXiv:hep-th/0402174

[58] Brett McInnes, Quintessential Maldacena-Maoz Cosmologies, JHEP 0404 (2004) 036, arXiv:hep-th/0403104

[59] Brett McInnes, Answering a Basic Objection to Bang/Crunch Holography, JHEP 10(2004)018, arXiv hep-th/0407189

[60] Jan de Boer, Liat Maoz, Asad Naqvi, Some Aspects of the AdS/CFT Correspondence, arXiv:hep-th/0407212

[61] M. Kleban, M. Porrati, R. Rabadan, Stability in Asymptotically AdS Spaces, arXiv:hep-th/0409242 
[62] Brett McInnes, Inflation, Large Branes, and the Shape of Space, Nucl. Phys. B709 (2005) 213, arXiv hep-th/0410115

[63] W. Fischler, S. Paban, M. Zanic, The Energy Density of "Wound" Fields in a Toroidal Universe, JHEP 0410 (2004) 041, arXiv:astro-ph/0407349

[64] Emilio Elizalde, Cosmology: Techniques and Observations, arXivigr-qc/0409076

[65] Ruth Durrer, Martin Kunz, Mairi Sakellariadou, Why do we live in 3+1 dimensions?, arXiv:hep-th/0501163

[66] Ya.B. Zeldovich, Alexei A. Starobinsky, Quantum Creation of a Universe in a Nontrivial Topology, Sov.Astron.Lett. 10 (1984) 135

[67] Jorma Louko, Peter J. Ruback, Spatially flat quantum cosmology, Class.Quant.Grav. 8 (1991) 91

[68] D. H. Coule, Jerome Martin, Quantum Cosmology and Open Universes, Phys.Rev. D61 (2000) 063501, arXiv:gr-qc/9905056

[69] D.H. Coule, Quantum Cosmological Models, arXiv:gr-qc/0412026

[70] Andrei Linde, Creation of a Compact Topologically Nontrivial Inflationary Universe, JCAP 0410 (2004) 004, arXiv:hep-th/0408164

[71] Damien A. Easson, Brane Gases on K3 and Calabi-Yau Manifolds, Int.J.Mod.Phys. A18 (2003) 4295, arXiv:hep-th/0110225

[72] R. Easther, B. R. Greene and M. G. Jackson, Cosmological string gas on orbifolds, Phys. Rev. D 66 (2002) 023502, arXiv:hep-th/0204099

[73] John Horton Conway, Juan Pablo Rossetti, Describing the platycosms, arXiv:math.DG/0311476

[74] A. Kehagias, K. Tamvakis, Box Compactification and Supersymmetry Breaking, Phys.Lett. B603 (2004) 249, arXiv hep-th/0403029

[75] Alain Riazuelo, Jeffrey Weeks, Jean-Philippe Uzan, Roland Lehoucq, Jean-Pierre Luminet, Cosmic microwave background anisotropies in multi-connected flat spaces, Phys.Rev. D69 (2004) 103518, arXiv:astro-ph/0311314

[76] Jean-Pierre Luminet, The Shape of Space after WMAP Data, arXiv:astro-ph/0501189

[77] Parampreet Singh, Effective State Metamorphosis in Semi-Classical Loop Quantum Cosmology, arXiv:gr-qc/0502086

[78] Brett McInnes, De Sitter and Schwarzschild-De Sitter According to Schwarzschild and De Sitter, JHEP 09(2003)009, arXiv:hep-th/0308022

[79] Jorma Louko, Robert B. Mann, Donald Marolf, Geons with spin and charge, arXiv:gr-qc/0412012 
[80] Raphael Bousso, Adventures in de Sitter space, arXiv:hep-th/0205177

[81] Ben Freivogel, Leonard Susskind, A Framework for the Landscape, Phys.Rev. D70 (2004) 126007, arXiv hep-th/0408133

[82] Brett McInnes, Orbifold Physics and de Sitter Spacetime, Nucl Phys B692(2004) 270, arXiv:hep-th/0311055

[83] Raphael Bousso, Cosmology and the S-matrix, arXiv:hep-th/0412197

[84] Frederic Leblond, Donald Marolf, Robert C. Myers, Tall tales from de Sitter space I: Renormalization group flows, JHEP 0206 (2002) 052, arXiv:hep-th/0202094

[85] Neil J. Cornish, David N. Spergel, Glenn D. Starkman, Eiichiro Komatsu, Constraining the Topology of the Universe, Phys.Rev.Lett. 92 (2004) 201302, arXiv:astro-ph/0310233

[86] Sean M. Carroll, Jennifer Chen, Spontaneous Inflation and the Origin of the Arrow of Time, arXiv:hep-th/0410270

[87] Raphael Bousso, The holographic principle, Rev.Mod.Phys. 74 (2002) 825, arXiv:hep-th/0203101

[88] Edward Witten, Anti De Sitter Space And Holography, Adv.Theor.Math.Phys. 2 (1998) 253, arXiv:hep-th/9802150

[89] Andreas Karch, Lisa Randall, Locally Localized Gravity, JHEP 0105 (2001) 008, arXiv:hep-th/0011156

[90] Michael Gutperle, Andrew Strominger, Spacelike Branes, JHEP 0204 (2002) 018 arXiv:hep-th/0202210

[91] Dumitru Astefanesei, Gregory C. Jones, S-branes and (Anti-)Bubbles in (A)dS Space, arXiv:hep-th/0502162

[92] Brett McInnes, in preparation.

[93] Joel K. Erickson, Daniel H. Wesley, Paul J. Steinhardt, Neil Turok, Kasner and Mixmaster behavior in universes with equation of state $\mathrm{w} \geq 1$, Phys.Rev. D69 (2004) 063514, arXiv:hep-th/0312009

[94] V. Bozza, G. Veneziano, Scalar perturbations in regular two-component bouncing cosmologies, arXiv:hep-th/0502047

[95] I.Ya. Aref'eva, A.S. Koshelev, S.Yu. Vernov, Exactly Solvable SFT Inspired Phantom Model, arXiv:astro-ph/0412619

[96] Gary Felder, Andrei Frolov, Lev Kofman, Andrei Linde, Cosmology With Negative Potentials, Phys.Rev. D66 (2002) 023507, arXiv hep-th/0202017

[97] Yun Wang, Jan Michael Kratochvil, Andrei Linde, Marina Shmakova, Current Observational Constraints on Cosmic Doomsday, JCAP 0412 (2004) 006, arXiv astro-ph/0409264 